\title{
Spectroscopic Studies of Charged Excitons
}

\author{
P. KOSSACKI \\ Institute of Experimental Physics, Warsaw University \\ Hoża 69, 00-681 Warszawa, Poland
}

\begin{abstract}
A brief review is given of optical experiments related to creation and recombination of charged excitons. Their results are analyzed in terms of a simple three-particle free trion model. It is shown that the simple model explains well such results as temperature variation of lifetime and absorption intensity or photoluminescence line shape and a decrease in absorption oscillator strength per carrier with increasing carrier concentration. The range of applicability of the simple model is examined and found to be limited by two factors: disorder-induced localization and many-body interactions occurring and high carrier sheet concentrations (of order of $10^{11} \mathrm{~cm}^{-2}$ ).
\end{abstract}

PACS numbers: 73.20.Mf, 78.40.Fy, 78.55.Et, 78.47.+p, 78.67.De

\section{Introduction}

The existence of charged excitons in semiconductors was predicted long ago (1958) by Lampert [1]. However, the small binding energy of the additional carrier makes the observation of these charged excitons in bulk material very difficult and possible only in extremely pure crystals [2, 3]. In $2 \mathrm{D}$ structures the binding energy is strongly enhanced and often charged excitons dominate the optical spectra of semiconductor quantum wells containing $2 \mathrm{D}$-carrier gas. The first identification of negatively charged excitons $\left(X^{-}\right)$in quantum wells was done by Kheng et al. [4]. This observation was rapidly followed by experimental evidence for both kinds of excitons in III-V and II-VI 2D systems [5-9]. Since then optical properties of such complexes have been intensively studied by means of several spectroscopic techniques. After the first controversy related to proper identification of the charged excitons, several important questions related to their properties were addressed. For example, detailed studies were performed on the selection rules $[10,30]$, dynamics [5, 11, 16], role of the carrier gas localization [12-14], localization of charged exciton in quantum well (QW) [14-16], the stability of 
the complex in high magnetic field $[17,18]$ and interaction between neutral and charged exciton states $[19,20]$.

We wish to discuss here selected problems related to optical properties of charged excitons in zero magnetic field. They will be illustrated by experimental results obtained on modulation doped CdTe-based quantum wells. In particular we plan to present a complete description of the oscillator strength in terms of a free three-particle charged exciton model. We will discuss the variation of radiative lifetime of the charged exciton with temperature and carrier concentration. It will be demonstrated that the experimentally observed linear increase in lifetime with temperature is predicted for delocalized trions for which the radiative recombination can take place with a specific strongly $k$ dependent selection rule [21, 22]. The same model describes correctly the change of photoluminescence (PL) line shape with temperature as well as the variation of absorption and PL spectra with carrier concentration. The validity of the model can be limited by localization phenomena and/or many-body effects at high concentrations of the carrier gas. We plan to estimate the importance of those phenomena by studying the range of applicability of the simple model.

\section{Experimental}

For our charged exciton studies we selected modulation doped CdTe-based quantum wells. Such choice is determined by a good structural and spectroscopic quality of II-VI heterostructures as well as much larger dissociation energies than for equivalent GaAs-based samples [5,6]. The samples containing a single $8 \mathrm{~nm}$ wide $\mathrm{Cd}_{1-x} \mathrm{Mn}_{x} \mathrm{Te} \mathrm{QW}$ were grown by molecular beam epitaxy. Two different designs were used for observation of positively and negatively charged excitons $\left(X^{+}\right.$and $X^{-}$). In the first case the barriers were made of $\mathrm{Cd}_{1-y-z} \mathrm{Mg}_{y} \mathrm{Zn}_{z} \mathrm{Te}$, in which the $\mathrm{Mg}$ content $(\gamma=0.25 \div 0.28)$ determined the valence band offset, while the presence of $\mathrm{Zn}(z=0.08 \div 0.07)$ ensured a good lattice match to the $\mathrm{Cd}_{0.88} \mathrm{Zn}_{0.12}$ Te substrate. The barriers were doped by nitrogen at three positions: at the distance $20 \mathrm{~nm}$ from the QW on the surface side and, in order to pin the Fermi level by neutralizing interface or surface charges, at $100 \mathrm{~nm}$ on each side of the QW.

Such design of the heterostructure makes it possible to tune the hole concentration isothermally by varying the intensity of illumination by photons with energy greater than the band gap of the barrier material. The photo-holes generated in this way are partially trapped in the barriers while the photo-electrons migrate to the QW and recombine with the holes confined therein. The trapped holes tunnel more slowly to the QW. A competition between these two processes determines the steady-state hole concentration $p$ [23]. The efficiency of this process was calibrated in the high concentration range by measuring the Moss-Burstein shift (the distance between the PL line and the energy of the absorption edge) and 
was discussed in detail in [23]. It was possible to tune hole concentration in the range from $1.3 \times 10^{10} \mathrm{~cm}^{-2}$ to $1.6 \times 10^{11} \mathrm{~cm}^{-2}$.

The samples used for $X^{-}$observation, were one-side modulation doped $\mathrm{Cd}_{1-x} \mathrm{Mn}_{x} \mathrm{Te} / \mathrm{Cd}_{0.75} \mathrm{Mg}_{0.25} \mathrm{Te}$ heterostructures. The remote donor layer of iodine was situated $10 \mathrm{~nm}$ from the QW. Its thickness was changed within the same sample in four steps: no doping layer, 2, 5, or 10 monolayers labeled hereafter spot $A, B, C$, and $D$. This allowed us to tune the concentration of the electron gas in steps by selecting one of the four different spots on the sample. Additionally, on a given spot, the electron concentration can be increased further by illuminating the sample with light of energy higher than the energy gap of the barrier. The mechanism of this effect is based on the competition between the QW and surface states which can both trap carriers and is similar to one used in $p$-type samples. The maximum attainable electron concentration was $2 \times 10^{11} \mathrm{~cm}^{-2}$ on spot $D$.

The experiments were carried out in helium cryostats with samples mounted strain-free and immersed in pumped liquid helium. The magnetic field was generated by a superconducting magnet. Photoluminescence was excited by $\mathrm{Al}_{2} \mathrm{O}_{3}: \mathrm{Ti}$ lasers with a power less than $2 \mathrm{~mW} / \mathrm{cm}^{2}$ in CW experiments or by a picosecond tuneable $\mathrm{Al}_{2} \mathrm{O}_{3}$ : Ti laser with a 2 ps pulse width, a repetition rate of $80 \mathrm{MHz}$, and an averaged power density of less than $100 \mathrm{~mW} \mathrm{~cm}^{-2}$ in time-resolved ones. The signal was collected through a spectrometer by a CCD camera or a $2 \mathrm{D}$ streak camera with 10 ps resolution. PL excitation was tuned below the energy gap of the barriers, so that neither carrier concentration nor spin temperature was perturbed. Also light used in transmission or reflectivity experiments was filtered by appropriate red filters. An additional illumination, serving to change the hole or electron concentration, was produced by standard halogen lamps equipped with one blue and several grey filters.

\section{Optical transitions}

The optical transitions related to creation or annihilation of neutral excitons were extensively studied by many authors (see for example [24]). The main qualitative difference between neutral and charged exciton transitions is related to different initial state (in absorption) or final state (in emission). For neutral exciton this state is the state with no band carriers. Therefore, the conservation of momentum and small wave vector of photon lead to a very small range of exciton $k$-vectors which are optically active. In contrary, the final state of charged exciton recombination consists of a band carrier. Thus charged exciton with $k$-vectors larger than zero can also recombine radiatively as its momentum can be transferred to the remaining particle. Therefore, a larger population of charged excitons with higher $k$-vectors is involved in the optical transitions. However, the probability of transition depends on the overlap between the wave function of free carrier and that of the charged exciton. It decreases with increasing momentum of both particles. The probabilities of optical transitions were calculated first by Stébé et al. for 
absorption [25] and later by Esser et al. [21] for luminescence. In both cases, the transition probabilities were based on a matrix element between initial and final states of the same momentum. The calculations where done for three-particle free complexes (trions). The trion wave functions $\Psi_{T}$ were obtained by numerical solution of three-particle Schrödinger equation. The optical transition matrix element is then given by the following equation:

$$
M(k)=\int \mathrm{d} \rho_{1} \mathrm{~d} \rho_{2} \Psi_{T}\left(\rho_{1}, \rho_{2}\right) \exp \left(-\mathrm{i} k \rho_{2} \frac{M_{X}}{M_{T}}\right) \delta\left(\rho_{1}\right),
$$

where $k$ denotes total momentum of trion or carrier, $M_{X}$ and $M_{T}=M_{X}+m$ masses of neutral exciton and trion and $\rho_{1}, \rho_{2}$ relative positions of electrons and holes in trion. It was shown [21] that the dependence of the matrix element on the wave vector can be very well approximated by an exponential function: $|M(k)|^{2} \sim$ $\exp \left[-\varepsilon(k) / \varepsilon_{1}\right]$, where $\varepsilon(k)=\hbar^{2} k^{2} M_{X} / 2 m M_{T}$. The characteristic energy $\varepsilon_{1}$ was obtained numerically and was equal, for example, $2 \mathrm{meV}$ and $0.6 \mathrm{meV}$ for $X^{-}$and $X^{+}$states, respectively, in an $8 \mathrm{~nm}$ wide CdTe quantum well. This form of matrix element is very convenient to explain the results of several optical experiments. First of all, the photoluminescence intensity can be described by using Fermi golden rule and a certain thermal distribution of trion states. In most PL experiments, the excitation is done with reasonably small power and the population of trions is small enough to justify approximation of its Boltzmann distribution. Neglecting broadening mechanisms, the intensity of the PL line is thus given by

$$
I_{T}(\hbar \omega) \sim|M(k)|^{2} \exp \left(-\frac{\varepsilon}{k_{\mathrm{B}} T} \frac{m}{M_{X}}\right) \theta(\varepsilon),
$$

in which $\theta$ is the Heaviside step function. The photon energy $\hbar \omega$ is given via $\varepsilon=E_{T}(k=0)-\hbar \omega=\hbar^{2} k^{2} M_{X} / 2 m M_{T}$. Equation (2) shows that the shape of PL line is asymmetric and has an exponential tail on the low energy side. The length $E_{\text {tail }}$ of this tail can be extracted and is given by $1 / E_{\text {tail }}=\left(1 / k_{\mathrm{B}} T\right)\left(m / M_{X}\right)+1 / \varepsilon_{1}$. The first term due to Boltzmann distribution leads to a linear increase in $E_{\text {tail }}$ with temperature and dominates at low temperatures. At higher temperatures, as higher $X_{T} k$-states are increasingly populated, the optical matrix element sets the maximum decay constant of the spectral tail at $\varepsilon_{1}$. This is in clear contrast to Ref. [14], where the low energy tail is claimed to increase continuously with temperature. Experimentally such behavior can be observed in PL measured as a function of temperature [21, 22]. Example spectra obtained for $X^{-}$line in $\mathrm{CdTe} / \mathrm{Cd}_{0.75} \mathrm{Mg}_{0.25} \mathrm{Te}$ quantum well are presented in Fig. 1a. The dashed lines show predicted slopes of the tail at the highest and lowest temperature for which two individual components of Eq. (2) are dominant. Figure 1b shows good agreement of the temperature variation of the PL tail with the presented model. However, one should note here that a correct description of the whole PL line shape must involve homogeneous and inhomogeneous broadening. In the presented case the second one was dominant and the true line shape was described by a convolution of a Gausssian line with 

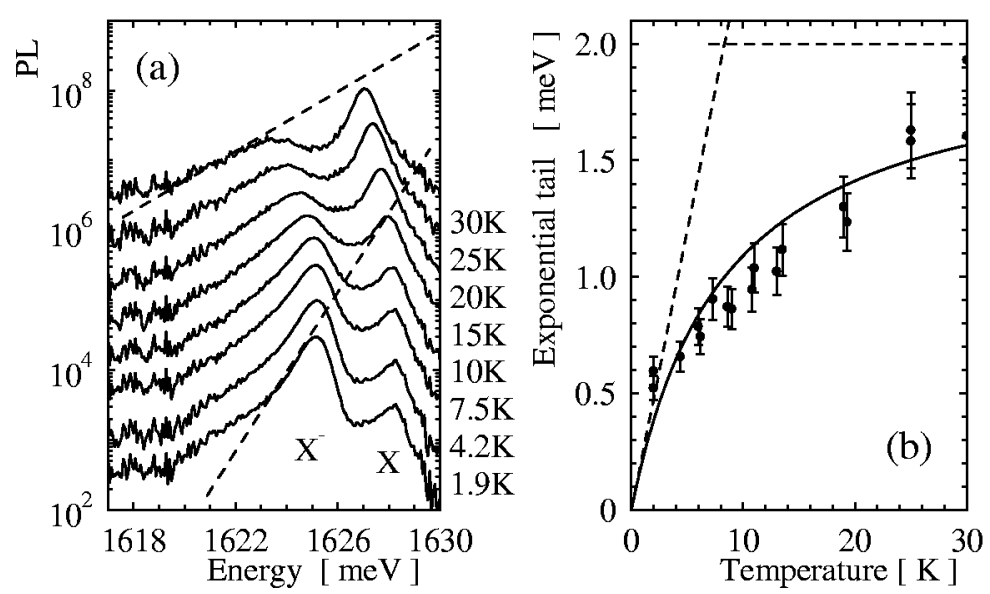

Fig. 1. (a) CW-photoluminescence spectra of $n$-doped $\mathrm{CdTe} / \mathrm{CdMgTe}$ quantum well at different temperatures. The dashed lines represent expected slopes of low energy tails at two selected temperatures. (b) Fitted values of the decay constant $E_{\text {tail }}$ of the $X^{-}$ exponential spectral tail as a function of temperature. The full line shows the calculated values of $E_{\text {tail }}$. The horizontal dashed line sets the value of $\varepsilon_{1}$, the other one shows the Boltzmann contribution of $E_{\text {tail }}$ [22].

that given by Eq. (2). The $X^{-}$line showed also a second tail in a lower energy with a significantly longer decay constant. This tail was due to localized states and will be discussed later.

The photoluminescence radiative decay time is inversely proportional to the average of the emission probabilities of all populated $k$-states. It can be obtained by integration of Eq. (2). Radiative decay time increases linearly with temperature as the maximum recombination probability is close to $k=0$ and the population of these states decreases with temperature. In particular, the analysis can be done analytically and it gives

$$
\tau_{T}=\frac{3 M_{X} \tau_{T 0}}{4 M_{T} E_{0}}\left(k_{\mathrm{B}} T+\varepsilon_{1} \frac{m}{M_{X}}+\frac{3}{5} \frac{M_{T}}{M_{X}} E_{0}\right),
$$

where the last term results from the finite momentum of photon (light cone effect) and can be neglected for temperatures higher than a few degrees of Kelvin. Parameters $E_{0}$ and $\tau_{T 0}$ are obtained by numerical integration with the use of material constants. The experimental check of this formula was done by time-resolved measurements of photoluminescence. In such experiment the radiative lifetime $\tau_{T}$ might be measured directly as a decay time of PL signal. However, in order to determine a correct value of the trion radiative lifetime, it is necessary to exclude any role of neutral exciton population. In particular, for low carrier concentrations and non resonant excitation the PL decay time is usually determined by decay of large population of neutral excitons. Such decay time is considerably longer 
than $\tau_{T}$. There are two experimental solutions: first is based on the use of samples with a high carrier concentration for which the formation time of charged exciton is short in comparison to $\tau_{T}$. In such case the $X$ population relaxes to charged exciton states and does not influence the decay time [27]. The second solution is to excite resonantly charged exciton line [28]. Additionally it is necessary to limit the temperature range by the value for which thermal dissociation appears (for $2 \mathrm{meV}$ dissociation energy and typical carrier concentrations it corresponds to about $30 \mathrm{~K}$ ). The second approach was used in [22]. The example $X^{-}$PL temporal profiles after pulse resonant excitation, are shown in Fig. 2a. The monoexponential decay was observed with decay time increasing with temperature. Figure $2 b$ shows that the calculated radiative lifetimes of $X^{-}$were in good agreement with the measurements. All deviations were within experimental accuracy. Only below $7 \mathrm{~K}$, the $X^{-}$lifetime deviated from the linear increase tending towards a constant value. This behavior is typical when localization influences the lifetime.
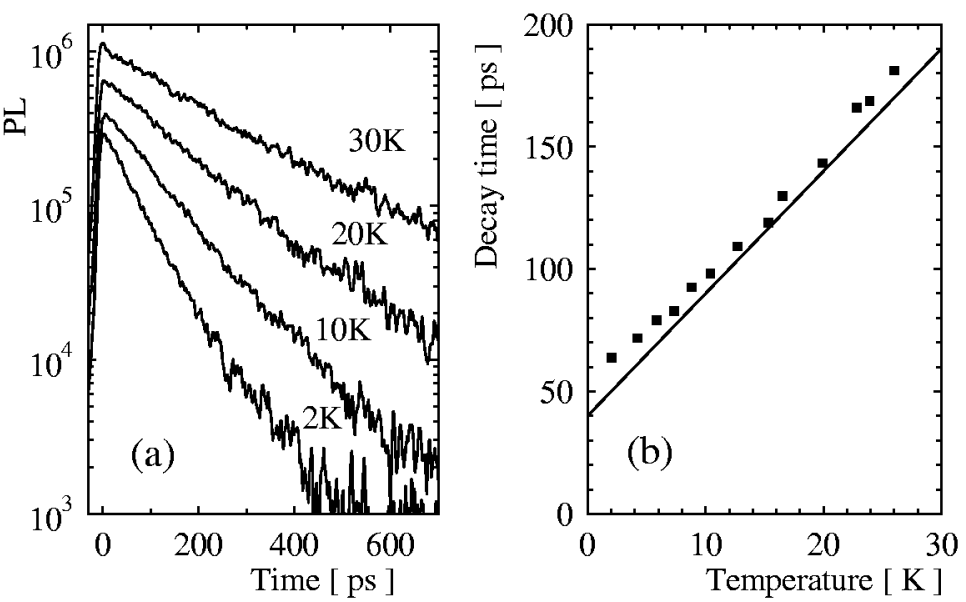

Fig. 2. (a) Time-resolved spectra of $X^{-}$in $n$-doped CdTe/CdMgTe quantum well for different temperatures. (b) Decay time of the $X^{-}$PL when exciting resonantly on $X^{-}$ as a function of temperature. The lines give the predictions of the model [22].

So far we have focused on the optical properties of emission due to charged exciton recombination. We will now link these properties to the absorption. The calculation of the absorption probability is very similar to Eq. (2). The Boltzmann thermal distribution of $X^{-}$is replaced by the thermal distribution of the electron which is now the initial state. For low electron concentrations, the electron gas is non degenerate and its thermal distribution follows also a Boltzmann distribution. It can be easily shown that the absorption probability is obtained by explicitly replacing $m$ by $M_{T}$ 


$$
I_{T}(\hbar \omega) \sim|M(k)|^{2} \exp \left(-\frac{\varepsilon}{k_{\mathrm{B}} T} \frac{M_{T}}{M_{X}}\right) \theta(\varepsilon) .
$$

This implies that the main difference between absorption and emission at low electron densities is due to the difference in masses between $X^{-}$or $X^{+}$and free carrier. Therefore, one can compare directly the temperature variation of the lifetime, and the absorption when the temperature axis is rescaled by $m / M_{T}$. The result obtained for reflectivity measurement on $X^{-}$sample is shown as a full line in Fig. 3b. It displays good agreement between the measured variations of the radiative lifetime and the absorption of $X^{-}$. This can be seen as another argument of the applicability of simple idealized model of free $X^{-}$. Here one should note that in principle also the absorption line is predicted to be asymmetric, with its low energy tail increasing with temperature. However, in realistic cases, this asymmetry is hardly accessible in experimental observations. For example the $\varepsilon_{1}$ parameter of $X^{-}$confined in QW of $8 \mathrm{~nm} \mathrm{CdTe}$ is equal to $2 \mathrm{meV}$ and the mass ratio is $m / M_{T}=0.25$. Then the maximum length of the tail at $T=30 \mathrm{~K}$ is only $0.9 \mathrm{meV}$ which is smaller than the normal line width at the same temperature [29].
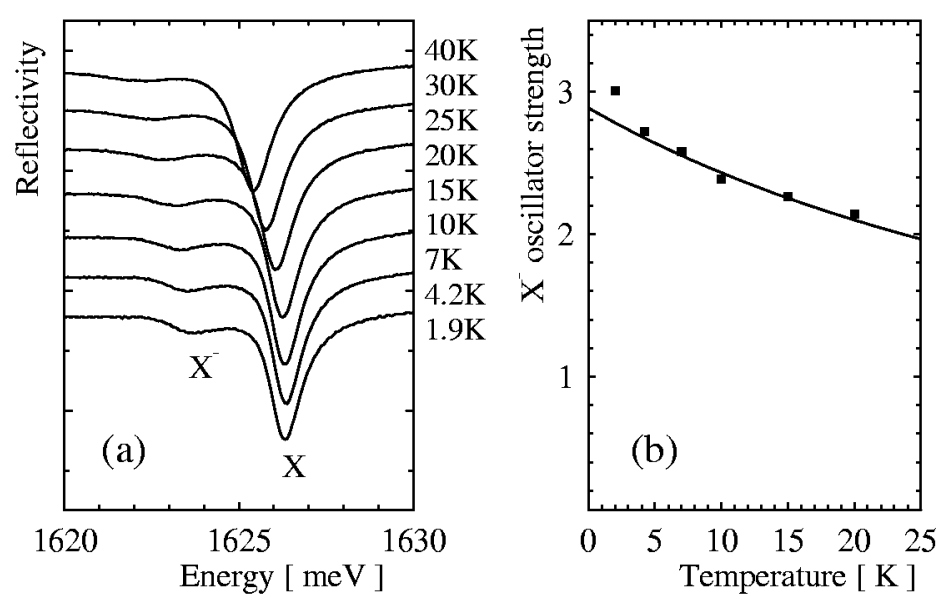

Fig. 3. (a) Normalized reflectivity spectra as a function of temperature for $n$-doped $\mathrm{CdTe} / \mathrm{CdMgTe}$ quantum well. (b) Extracted relative oscillator strength of $X^{-}$in arbitrary units (points) and comparison with the rescaled inverse trion lifetime (solid line).

Another test of the above model was done [27] by increasing the carrier concentration. Then the Boltzmann distribution of carriers cannot be applied. It has to be replaced by Fermi-Dirac distribution, which leads to the modification of Eq. (4). As a result, even at low temperature, the increasing carrier concentration results in populating states with higher $k$-vector. Therefore the probability of optical transition per carrier is reduced. Such experiment was done in transmission, for $X^{+}[27]$. The appropriate spectra and absorption oscillator strength per hole 

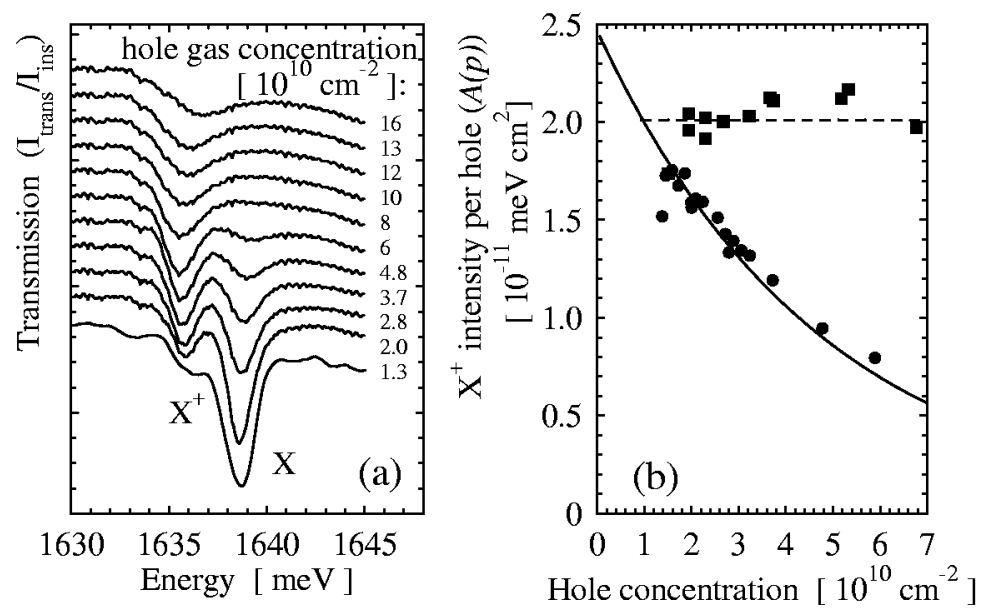

Fig. 4. (a) Transmission spectra for different hole gas concentrations as controlled by additional blue light illumination. (b) Integrated intensity of $X^{+}$transmission line per hole versus hole concentration $A(p)$ (squares) compared to the oscillator strength extracted from inverse trion lifetime (circles) [27]. The temperature was $1.3 \mathrm{~K}$. The lines represent predictions of the model described in the text.

$A(p)$ are presented in Fig. 4a and Fig. 4b (solid circles). The solid line in Fig. 4b was obtained numerically assuming Fermi-Dirac distribution at $T=2 \mathrm{~K}$ for the independently calculated parameter $\varepsilon_{1}=0.6 \mathrm{meV}$. It shows excellent agreement with the experimental data. It is interesting to point out that oscillator strength per carrier obtained from transmission measurement can be compared to radiative PL lifetime. The quantitative comparison of both kinds of data (PL and absorption) can be done using an atomic-like formula [24, 30], which has been successfully applied for excitons bound to impurities. Such an approach leads to the following relation between lifetime and absorption:

$$
\frac{\hbar}{\tau}=\frac{n^{2} \omega^{2}}{3 \pi^{2} c^{2}} A(p)
$$

where $n$ is the refractive index, $c$ - the velocity of light, and $\omega$ - the frequency. The expected oscillator strength $A(p)$ extracted from PL lifetime measurements [27] and taking into account the effect of the cap layer [23, 31] is shown by solid circles in Fig. 4b. One notices good agreement between both experiments for vanishing hole density, while for the higher ones the difference increases and reaches a factor of two for $6 \times 10^{10} \mathrm{~cm}^{-2}$. This difference is relatively obvious since in the PL experiment, in contrary to absorption, always very few initial states are populated. Therefore, the charged excitons, for all hole concentrations, might thermalize to states of $k$-vector close to zero.

Summarizing, a decrease in trion oscillator strength is always observed when initial states of higher $k$-vector are populated. It might be caused by an increase 
in temperature or by Pauli exclusion rule when increasing carrier concentration. The same effect is observed in absorption and in emission experiments. This is in contrast to the behavior of neutral exciton for which the radiative decay time increases with temperature but the absorption oscillator strength remains almost constant.

\section{Limits of free trion approach}

In the above discussion, it was assumed that charged excitons are delocalized three-particle complexes. There are two most obvious limits of such approach. The first one is constituted by many-body interactions in dense carrier systems. At high concentrations we must see the charged exciton rather as a collective state of the carrier sea induced by the presence of a carrier of opposite sign. This effect can be seen in Fig. 4, where spectra with clearly distinguishable $X$ and $X^{+}$lines are visible only up to about $6 \times 10^{10} \mathrm{~cm}^{-2}$. Therefore, we limit the range of the hole concentrations in Fig. 4b to about that value. In the following discussion we would like to focus on a second limitation of the simple model, i.e., a localization in any kind of disorder. There are two limits of the localization. If the localization length is small, the wave function of charged exciton is significantly perturbed. In that case, the model of free trion discussed before, cannot be applied. It is necessary to perform three-particle calculations with a defined confining potential. If the confining potential is Coulomb one, the problem reassembles rather the problem of $\mathrm{D}^{0} \mathrm{X}$ or $\mathrm{A}^{0} \mathrm{X}$ complexes. Other limit, is the localization in shallow, long-range fluctuations of potential. Then the localization range is long in comparison to the charged exciton size and charged exciton might be treated as a quasiparticle of a

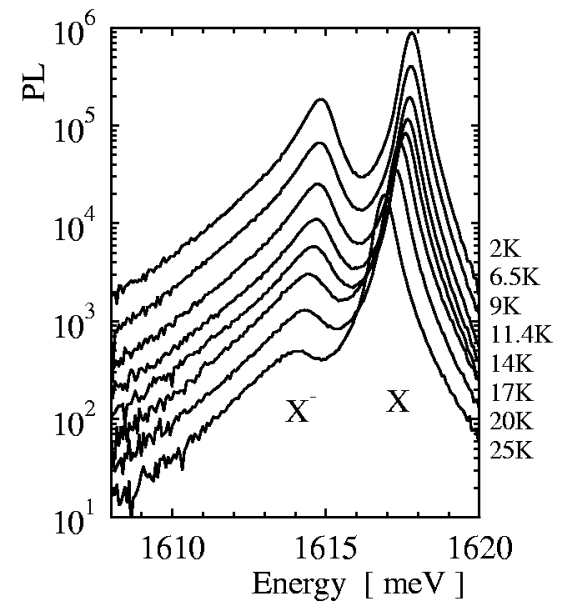

Fig. 5. Low excitation CW-photoluminescence spectra of $n$-doped $\mathrm{CdTe} / \mathrm{CdMgTe}$ quantum well with a very low electron concentration at different temperatures. 
given center of mass motion. The wave function of such localized state, is composed of trion three-particle wave functions ofdifferent $k$-vectors. As localization length is smaller, the higher $k$-vectors are more important. In order to describe optical transition, it is possible to employ a similar approach as for thermal distribution of free trion states. It leads to a formula equivalent to Eq. (2). Therefore, the charged exciton localization results in asymmetry of PL line with a low energy tail even at lowest temperatures. The PL spectra showing such tails were observed for several samples containing $X^{-}$. Example spectra are presented in Fig. 5. The length of low energy tails is limited by the value of the $\varepsilon_{1}$ parameter, the same way as it was limited for temperature dependence of PL line shape of free trion origin. This explains why the tails of localized states do not change too much with temperature in Fig. 5.

The increasing contribution of higher $k$-vector components, leads to a decrease in optical transition probability and an increase in PL-decay time. This explains the deviation from the linear increase, of $X^{-}$lifetime, below $7 \mathrm{~K}$ (Fig. 2b).

\section{Conclusions}

Selected problems related to optical properties of charged excitons were discussed. A complete model is presented of delocalized charged exciton for which the radiative transition can take place with a specific strongly $k$ dependent selection rule. This model describes correctly the variation of radiative lifetime of the charged exciton with temperature as well as the asymmetric PL line shape and its variation with temperature. The same model describes precisely the change of the absorption with temperature and carrier concentration. The model based on similar assumptions, can be also employed in description of the shallow localization of charged exciton center of mass. The presented analysis indicates the application limits of the model, caused by localization and/or many-body interactions.

\section{Acknowledgments}

I thank my co-workers: V. Ciullin, A. Esser, J. Cibert, M. Kutrowski, W. Maslana, S. Tatarenko, D. Ferrand, T. Wojtowicz, B. Deveaud, and J.A. Gaj. The work was partially supported by the State Committee for Scientific Research (grants 5P03B02320 and 2P03B09418) and by the Polish-French collaboration program "Polonium" (03307ZG).

\section{References}

[1] M.A. Lampert, Phys. Rev. Lett. 1, 450 (1958).

[2] G.A. Thomas, T.M. Rice, Solid State Commun. 23, 359 (1977)

[3] T. Kawabata, K. Muro, S. Narita, Solid State Commun. 23, 267 (1977). 
[4] K. Kheng, R.T. Cox, Y. Merle d'Aubigné, Franck Bassani, K. Saminadayar, S. Tatarenko, Phys. Rev. Lett. 71, 1752 (1993).

[5] G. Finkelstein, H. Shtrikman, I. Bar-Joseph, Phys. Rev. Lett. 74, 976 (1995).

[6] A.J. Shields, J.L. Osborne, M.Y. Simmons, M. Pepper, D.A. Ritchie, Phys. Rev. B 52, R5523 (1995)

[7] A. Haury, A. Arnoult, V.A. Chitta, J. Cibert, Y. Merle d'Aubigné, S. Tatarenko, A. Wasiela, Superlattices Microstruct. 23, 1097 (1998).

[8] T. Wojtowicz, M. Kutrowski, G. Karczewski, J. Kossut, F.J. Teran, M. Potemski, Phys. Rev. B 59, R10437 (1999); T. Wojtowicz, M. Kutrowski, G. Karczewski, J. Kossut, Appl. Phys. Lett. 73, 1379 (1998).

[9] G.V. Astakhov, D.R. Yakovlev, V.P. Kochereshko, W. Ossau, J. Nürnberger, W. Faschinger, G. Landwehr, Phys. Rev. B 60, R8485 (1999).

[10] S. Lovisa, R.T. Cox, N. Magnea, K. Saminadayar, Phys. Rev. B 56, R12787 (1997).

[11] E. Vanelle, M. Paillard, X. Marie, T. Amand, P. Gilliot, D. Brinkmann, R. Lévy, J. Cibert, S. Tatarenko, Phys. Rev. B 62, 2696 (2000).

[12] K. Kheng, R.T. Cox, T. Baron, K. Saminadayar, S. Tatarenko, J. Cryst. Growth 159, 443 (1996).

[13] G. Eytan, Y. Yayon, M. Rappaport, H. Shtrikman, I. Bar-Joseph, Phys. Rev. Lett. 81, 1666 (1998).

[14] D. Brinkmann, J. Kudrna, P. Gilliot, B. Hönerlage, A. Arnoult, J. Cibert, S. Tatarenko, Phys. Rev. B 60, 4474 (1999).

[15] H.P. Wagner, H.-P. Tranitz, R. Schuster, Phys. Rev. B 60, 15542 (1999).

[16] H.W. Yoon, A. Ron, M.D. Sturge, L.N. Pfeiffer, Solid State Commun. 100, 743 (1996).

[17] A.J. Shields, M. Pepper, M.Y. Simmons, D.A. Ritchie, Phys. Rev. B 52, 7841 (1995).

[18] S.A. Crooker, E. Johnston-Halperin, D.D. Awschalom, R. Knobel, N. Samarth, Phys. Rev. B 61, R16307 (2000).

[19] P. Gilliot, D. Brinkmann, J. Kudrna, O. Crégut, R. Lévy, A. Arnoult, J. Cibert, S. Tatarenko, Phys. Rev. B 60, 5797 (1999).

[20] T. Brunhes, R. André, A. Arnoult, J. Cibert, A. Wasiela, Phys. Rev. B 60, 11568 (1999).

[21] A. Esser, E. Runge, R. Zimmermann, W. Langbein, Phys. Rev. B 62, 8232 (2000).

[22] V. Ciulin, P. Kossacki, S. Haacke, J.-D. Ganiére, B. Deveaud, A. Esser, M. Kutrowski, T. Wojtowicz, Phys. Rev. B 62, R16310 (2000).

[23] P. Kossacki, J. Cibert, D. Ferrand, Y. Merle d'Aubigné, A. Arnoult, A. Wasiela, S. Tatarenko, J.A. Gaj, Phys. Rev. B 60, 16018 (1999).

[24] L.C. Andreani, in: Confined Electrons and Photons, New Physics and Applications, Eds. E. Burnstein, C. Weisbuch, Plenum Press, New York 1994.

[25] B. Stébé, E. Feddi, A. Ainane, F. Dujardin, Phys. Rev. B 58, 9926 (1998) and references therein. 
[26] O.V. Volkov, V.E. Zhitomirskii, I.V. Kukushkin, V.E. Bisti, K. von Klitzing, K. Eberl, JETP Lett. 66, 766 (1997).

[27] P. Kossacki, V. Ciulin, J. Cibert, Y. Merle d'Aubigné, A. Arnoult, C. Bourgognon, A. Wasiela, S. Tatarenko, J.-L. Staehli, J.-D. Ganiere, B. Deveaud, J.A. Gaj, J. Cryst. Growth 214/215, 837 (2000).

[28] G. Finkelstein, V. Umansky, I. Bar-Joseph, V. Ciulin, S. Haacke, J.-D. Ganiére, B. Deveaud, Phys. Rev. B 58, 12637 (1998).

[29] E.J. Mayer, N.T. Pelekanos, J. Kuhl, N. Magnea, H. Mariette, Phys. Rev. B 51, 17263 (1995).

[30] K. Kheng, Ann. Phys. (France) 20, C2-229 (1995).

[31] Y. Merle d'Aubigné, A. Wasiela, H. Mariette, T. Dietl, Phys. Rev. B 54, 14003 (1996). 